\title{
ALGUNOS ASPECTOS ECONÓMICOS DE LA RESPONSABILIDAD EXTRACONTRACTUAL DEL ESTADO*
}

\section{RAÚL LETELIER WARTENBERG**}

RESUMEN: El presente trabajo explora la aplicación del análisis económico al campo de la responsabilidad extracontractual del Estado. Luego de aceptar su utilización como herramienta general tendiente a la optimización de los costos sociales de evitar accidentes se enfoca en algunas particularidades de la responsabilidad pública que debiesen ser consideradas en cualquier análisis de este tipo.

PALABRAS CLAVE: Responsabilidad extracontractual - responsabilidad Estado - aspectos económicos - responsabilidad

\section{SOME ECONOMIC ASPECTS OF THE EXTRA CONTRACTUAL LIABILITY OF STATE}

ABSTRACT: This paper is about the application of economic analysis to the field of extra contractual liability of the State. After accepting its use as a general tool aimed at optimizing the social costs to avoid accidents, it is focused on some peculiarities of public liability that must be considered in any analysis of this kind.

KEY WORDS: Extra contractual liability - Liability of the State - Economic aspects - Liability.

Sumario: 1) Introducción. 2) La maximización de la riqueza y la minimización de los costos sociales de los accidentes. 2.1) El problema del gasto obligatorio. 2.2) El problema de los bienes institucionales. 2.3) El problema de los gastos de administración. 2.4) El problema de los pagos. 2.5) El proble-

\footnotetext{
* Fecha de recepción: 22 de septiembre de 2012.

Fecha de aceptación: 6 de marzo de 2013.

** Doctor en Derecho, Profesor de Derecho Administrativo de la Facultad de Derecho de la Universidad Alberto Hurtado. Correo electrónico: rletelier@uahurtado.cl. Este trabajo forma parte del proyecto de investigación Fondecyt No 11090088 titulado Philosophical Foundation of Governmental Liability del que el autor es investigador principal.
} 
ma del mercado como foco de análisis. 2.6) El problema de la legalidad. 3) Conclusiones. 4) Bibliografía.

\section{1) INTRODUCCIÓN}

Dos son los fines que bien podrían considerarse "últimos" en la responsabilidad extracontractual ${ }^{1}$. Por un lado, sus reglas pretenden maximizar riqueza ${ }^{2}, y$, por otro, pretenden condicionar la acción de las personas hacia ciertos modelos de conductas socialmente estimados como buenos o correctos $^{3}$. Para alcanzar estos objetivos, las normas de responsabilidad se sirven de un fuerte instrumento jurídico, este es, imponer una obligación coercible en el patrimonio de una persona de tener que transferir una cantidad de su patrimonio a otra. Esta imposición es desde la perspectiva de quien debe desembolsarla una verdadera sanción. Por otra parte, si el Derecho ordena la imposición de aquella sanción bien puede decirse también que la persona a quien debe pagársele tiene un "derecho" a que ella se haga efectiva. Derechos y obligaciones, son así, las herramientas jurídicas de que se vale instrumentalmente la responsabilidad para alcanzar los fines ya indicados.

Desde esta perspectiva, entonces, ¿por qué debe una persona pagar a otra el daño generado? Debe pagar porque de conservar aquella cantidad de dinero, la riqueza no estaría siendo maximizada y se estaría avalando un modelo de conducta humana no considerado socialmente como justo o correcto ${ }^{4}$.

Como puede percibirse entonces, un aspecto económico y otro moral se disputan la explicación acerca de los fundamentos de la responsabilidad extracontractual. El presente trabajo pretende tomar en consideración el aspecto económico y confrontarlo con la responsabilidad extracontractual del Estado. Todo ello con el objeto de detectar si los vectores de intereses que regulan una y otra aproximación son los mismos

1 Estos fines pueden también descomponerse en otros para un análisis más directo. Cane, P. (2006) Antiyah's accidents, compensation and the law, 7a edición. New York: Cambridge, p. 408. Sobre un análisis principalmente económico. Cooter, R. (1991). "Economic Theories of Legal Liability". The Journal of Economic Perspectives, Vol. 5, No 3, p. 11-30; Faure, M. (2009) Tort Law and Economics. Massachusetts: Elgar.

2 Posner, R. (1995). "Wealth Maximization and Tort Law: A Philosophical Inquiry". En Owen, D. G. (editor): Philosophical Foundations of Tort Law. New York: Oxford University Press, p. 99 y ss.

3 Cane, P. (2002). "Tort Law as Regulation". Common Law World Review, No 31, p. 305 y ss. y Cane, P. (1997) The anatomy of tort law. Oxford: Hart, p. 28 y ss.

$4 \quad$ Una versión de complementación de estos dos objetivos puede verse en GeistFeld, M. (2001). "Economic, Moral Philosophy, and the Positive Analysis of Tort Law". En Postema, G.J. (editor): Philosophy and the law of torts. New York: Cambridge, p. $250 \mathrm{y}$ ss. 
y si, por ello, la mecánica de incentivos y cargas de la responsabilidad civil puede trasladarse sin problemas a la esfera pública.

\section{2) LA MAXIMIZACIÓN DE LA RIQUEZA Y LA MINIMIZACIÓN DE LOS COSTOS SOCIALES DE LOS ACCIDENTES}

La responsabilidad extracontractual es uno de entre varios instrumentos que pueden ser utilizados por el Estado para modular la actividad social $^{5} \mathrm{y}$, por sobre todo, producir en la sociedad aquella referida maximización de la riqueza general ${ }^{6}$. Aunque este último concepto no resulte del todo claro, podemos aceptar como bastante razonable el concepto de Posner que entiende por riqueza "el valor total de todos los bienes y servicios "económicos" y "no económicos"”, riqueza que se maximiza "cuando todos los bienes y servicios, en la medida en que esto sea posible, sean asignados a sus usos más rentables" 7 .

En el caso de la responsabilidad extracontractual, los sujetos cuyas riquezas debemos maximizar son focalizadamente aquel que sufre un daño y aquel que lo provoca. Ambos están insertos en un esquema de maximización de riqueza. Aquel que sufre el daño obviamente, pues su pérdida es fácilmente cuantificable pero también aquel que la provoca pues la sanción que se le impondrá no es sino una forma de hacerle internalizar aquel daño por no haber gastado dinero en haber tomado las medidas de precaución necesarias y razonables para evitarlo. No obstante, como también sabemos, no es en lo absoluto exigible evitar accidentes a cualquier costo $^{8}$. Habrá, entonces, un gasto en precaución que maximice la riqueza general.

Como puede percibirse de lo anterior y como se constata por lo demás en la vida diaria, el daño puede ser entendido como una función de la precaución. Más específicamente, puede sostenerse que la probabilidad de la ocurrencia de un daño disminuye mientras mayor sea la precaución que se adopte. Que sea una función quiere decir que es posible representar los cambios en una variable (la probabilidad de que acaezca un daño)

\footnotetext{
5 Ogus, A. (2007). "Shifts in Governance for Compensation to Damage: A Framework for Analysis”. En Van Boom, W.H. y Faure, M. (editores): Shifts en Compensation between Private and Public Systems. Wien: Springer, p. 33 y ss.

6 OGUS (2007) 33.

7 Posner (1995) 99. La recepción de esta idea por alguna jurisprudencia comparada en BARnes, D. W. y Stout, L. A. (1992) The Economic Analysis of Tort Law. St. Paul: West. Para una percepción más general del análisis económico del derecho de daños vid. Kuperberg, M. y Beitz, C. (1983) Law, Economics and Philosophy. New Jersey: Rowman \& Allanheld.

8 Calabresi, G. (2005). "El costo de los accidentes". En Rosenkrantz, C. (editor): La responsabilidad extracontractual. Barcelona: Gedisa, p. 85.
} 
mediante las variaciones de otra variable (la cantidad de medidas de precaución adoptadas).

Como puede apreciarse en la figura 1 la probabilidad de la ocurrencia de un daño, $[\mathrm{p}(\mathrm{x}) \mathrm{A}$, en donde $\mathrm{A}$ es el valor del daño multiplicado por su probabilidad de ocurrencia] decrece si se adoptan mayores medidas de cuidado. Sin embargo, como también puede apreciarse en la línea wx [que representa el costo de una unidad de precaución (w) multiplicada por la cantidad de medidas de precaución adoptadas, $\mathrm{x}$ ], el aumento en medidas de precaución acrecienta naturalmente el gasto en ellas 9 . En pocas palabras, ser cuidadoso cuesta dinero.

FIG. 1

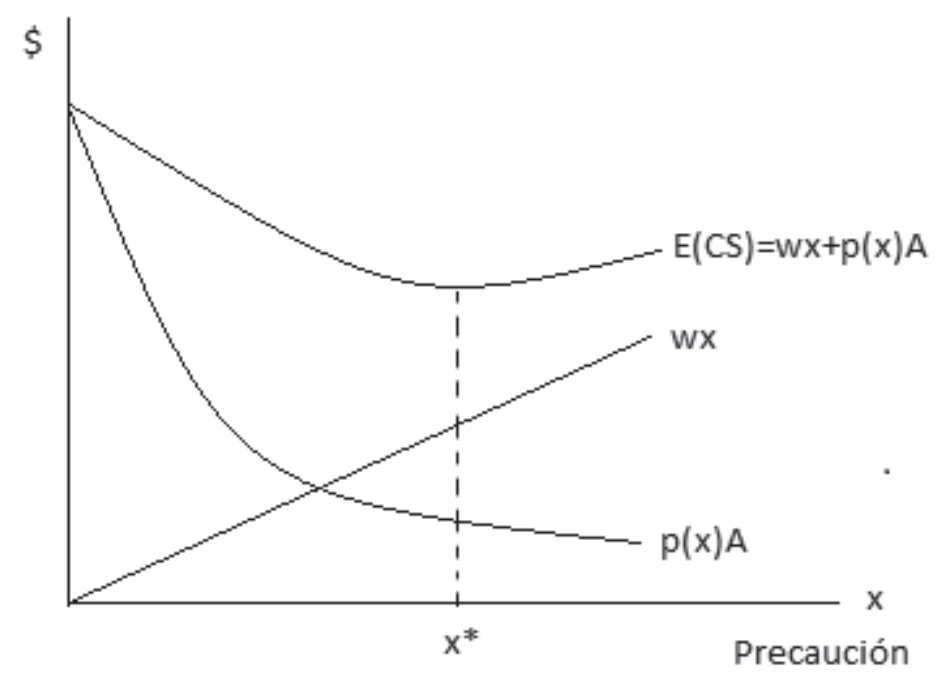

Si sumamos los costos de los daños esperados $(\mathrm{p}(\mathrm{x}) \mathrm{A})$ a lo gastado en precaución (wx) obtenemos una curva que representa esa sumatoria y a la que llamamos Costo Social (CS). Dicha curva posee una parte descendente, una base y una fase ascendente reflejando la existencia de un punto de minimización de aquellos costos sociales y que localizamos en el punto $\mathrm{x}^{*}$ reflejando el número de medidas de precaución que minimizando aquellos costos sociales maximiza también la riqueza de aquel que debe invertir en esas medidas ${ }^{10}$.

\footnotetext{
9 Cooter, R. (2000) Law and Economics, 3a edición. Berkeley: Addison Wesley Longman, p. 301. 10 Una primera etapa de este desarrollo económico puede encontrarse en la ya célebre fórmula de Learned Hand. Aвraham, K. S. (2007) The Forms and Functions of Tort Law, $3^{\text {a }}$ edición. New York: Foundation Press, p. 62 y ss.
} 
Este punto de maximización genera claros incentivos en las personas, conduciendo a que exista un mayor número de actividades beneficiosas para la vida a un costo razonable. Tal como explica Calabresi "quienes realizan actividades relativamente peligrosas a un precio que no refleja el costo de los accidentes, se volcarán a actividades más seguras si los costos de los accidentes se reflejaran efectivamente en el precio que tienen que pagar por dichas actividades" 11 .

Este es el análisis económico que usualmente suele asociarse a los denominados ilícitos culposos civiles. En tanto su objetivo es maximizar riquezas, opera generando una disuasión de conductas que atenten contra aquella optimización y fomentan el desarrollo más o menos seguro de acciones humanas.

Creo que este modelo puede ser en esencia traspuesto al ámbito de la responsabilidad extracontractual del Estado. Y esto es así pues su lógica interna puede ser aplicada a muchos escenarios donde las probabilidad de un daño dependen de las acciones u omisiones de aquel que se ubique en posición de ser un potencial dañador. Así las cosas, una representación en clave maximizadora de beneficios puede ser "en principio" de mucha ayuda para las decisiones compensatorias adoptadas en sede pública.

Sin embargo, existen diversas particularidades en el ámbito de la responsabilidad pública que escapan de un razonamiento de costo/beneficio como el que hemos mostrado hasta el momento y que obliga a replantearse o al menos matizar la extensión de aquella aplicación. Veamos algunos de estos conflictos:

\section{1) El PROBleMA DEL gaSto ObLigatorio}

En primer lugar -y esta es una de las premisas a las que se enfrenta cualquier evaluación de gasto público- la manera de calcular la eficiencia asignada a los recursos públicos no pasa necesariamente por un cálculo de costo y beneficio que maximice las utilidades netas de una determinada acción sino más bien por uno de "costo-efectividad". En resumen, y tal como lo explica acertadamente Belli, existen servicios que deben ser necesariamente prestados sin posibilidad de decidir acerca de su mantención o extinción. La evaluación económica, en efecto, solo pretende hacer que cada unidad monetaria logre la mayor accesibilidad al servicio, o más bien, su mayor efectividad posible ${ }^{12}$.

En estas condiciones, la utilidad marginal de cada unidad gastada en precaución puede estar inspirada no ya solo en la búsqueda de una opti-

\footnotetext{
11 Calabresi (2005) 93.

12 Belli, P., et al. (2001) Economic Analysis of Investment Operations. Washington: World Bank, p. 2,78 .
} 
mización entre probabilidad de daño y costo sino que puede venir inspirada en una decisión previa de carácter político que ordena la provisión de un determinado servicio a la población, decisión esta que puede ser perfectamente ajena a una discusión de eficiencia.

En el mismo sentido, y puestos en un escenario de presupuesto limitado, la existencia de costos obligatorios puede hacer disminuir el gasto en medidas de precaución. Ello debe ser entonces considerado por nuestro análisis económico.

Por otra parte, el fin disuasorio de las condenas de responsabilidad puede generar una línea de fuerza inversa a aquella decisión previa de que tal o cual servicio público deba necesariamente prestarse. De ahí que no resulte tan claro aplicar el análisis económico que suele hacerse a las acciones u omisiones privadas cuando se debe "resistir" a aquella disuasión esperada por no permitirlo el propio sistema jurídico.

\section{2) EL PROBLEMA DE LOS BIENES INSTITUCIONALES}

Si nuestro análisis en la primera parte comparaba los gastos de precaución y los beneficios dados por la evitación del daño, esta matriz supone tener cierta claridad en los beneficios generados por la actividad. Ello no resulta ser tan sencillo en el ámbito público toda vez que al lado de una mayor accesibilidad de determinado servicio pueden también potenciarse diversos bienes públicos cuyos beneficios pueden ser difíciles de medir $^{13}$. Los casos de responsabilidad objetiva son un buen ejemplo de esta dificultad de calcular los beneficios toda vez que en este tipo de responsabilidad el cálculo de maximización de la riqueza supone evaluar necesariamente los beneficios directos de la actividad ${ }^{14}$.

La aplicación de un régimen de responsabilidad estricta al uso de armas de fuego, por ejemplo, bien podría ser analizado del siguiente modo: en tanto ese uso importa un aumento de los riesgos sociales de daños, su utilización solo será viable económicamente si los riesgos están muy reducidos (tengo solo un arma, bajo llave, en mi casa) o si los beneficios que obtengo por ese uso son cuantiosos (la uso para proteger un banco con mucho dinero dentro $)^{15}$.

Sin embargo, cuando existe también un uso institucional de las mismas armas que provee principalmente un bien público como la seguridad social no resulta tan fácil realizar ese cálculo. En efecto, la inexistencia de armamento institucional puede generar un nivel de riesgo absolutamente

13 Cohen, D. (1990). "Suing the State". University of Toronto Law Journal, No 40, p. 645.

14 Vandall, F. (1989) Strict Liability. Legal and Economic Analysis. Connecticut: Greenwood Press, p. 51.

15 Sobre esta idea de riesgos: Perry, S. (2007) “Risk, Harm, Interests and Rights”. En Lewens, T. (editor): Risk. Philosophical Perspectives. London: Routledge, p. 190 y ss. 
intolerable para la población. En este estado de cosas, el beneficio marginal de cada arma en términos de disminución de riesgos es muy alto en los primeros aumentos. Y ello es así aun cuando el ingreso de cada nueva arma genere en sí mismo un aumento de los niveles de riesgo por manejo negligente de ellas. En un punto $\mathrm{a}^{*}$ sin embargo se alcanza un nivel de riesgo óptimo $\left(\mathrm{r}^{*}\right)$ donde ya no es posible seguir disminuyendo el nivel de riesgo social. No obstante, el ingreso de una nueva unidad de armamento volverá a aumentar el nivel de riesgo toda vez que la probabilidad de un uso negligente ya no podrá ser compensada por el beneficio social en seguridad obtenido por las unidades anteriores al punto $\mathrm{a}^{*}$ tal como puede verse en la figura 2 .

\section{FIG. 2}

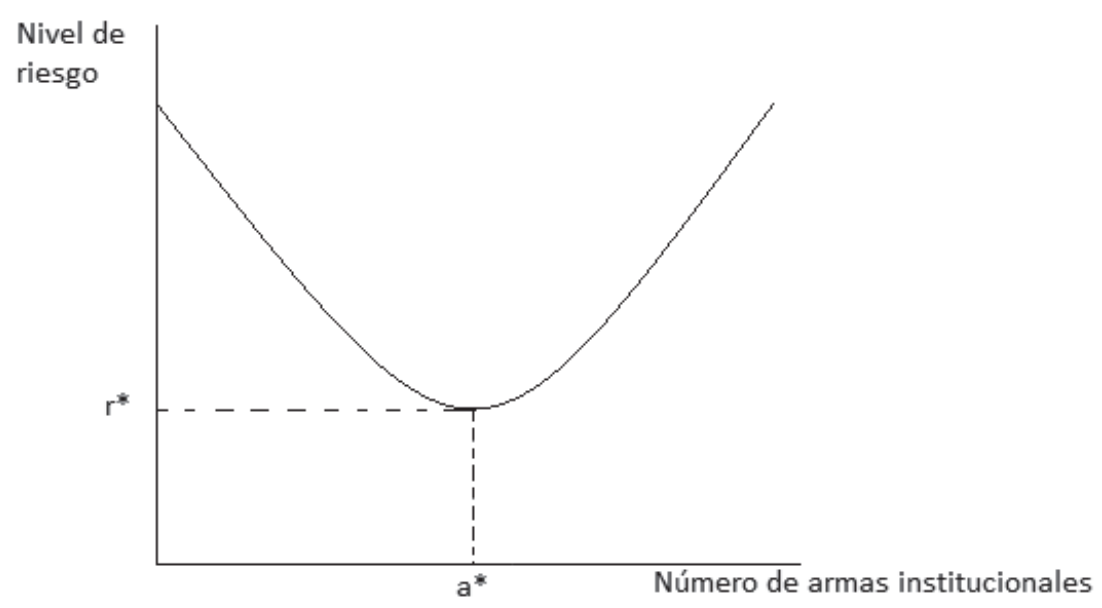

Esta realidad muestra la complejidad del análisis de beneficios en el caso de actividad pública y la existencia de beneficios dispersos, todo lo cual distorsiona la justificación económica de los casos de responsabilidad objetiva donde son precisamente aquellos beneficios del riesgo que se asume los que sirven de soporte a ese especial tipo de responsabilidad. Así, desde una óptica de riesgos no sería razonable fundar una responsabilidad objetiva basada en el riesgo creado por una unidad de armamento si esa unidad provee un beneficio institucional del cual nos aprovechamos todos, riesgo por lo demás que es inherente a la misma disminución de un riesgo social.

\section{3) El PROBLEMA DE los GASTOS DE ADMINISTRACióN}

Obtener una sentencia judicial que dé lugar a los dańos solicitados cuesta dinero. Debe invertirse en horas/hombre de un juez y de un grupo 
de funcionarios que analizarán el caso respectivo. Si lo que se pretende es maximizar la riqueza debe también considerarse la reducción de estos gastos de administración. El problema en nuestro caso es que tanto los gastos en precaución como estos gastos de administración recaen en la misma persona jurídica. En efecto, en tanto el aparato jurisdiccional es un órgano estatal los gastos en su mantención deben ser imputados al erario. En el modelo privado, los gastos de administración son contabilizados para optar u optimizar un determinado medio de compensación. En el ámbito público, por el contrario, es el costo global de esta administración la partida que debe ser analizada.

\section{4) El PROBlema de los PAgos}

En el ámbito privado, la forma de ejecutar la compensación se realiza a través del pago de la indemnización condenada. En una explicación que ya resulta tradicional y bajo la premisa que todos los bienes pueden ser intercambiados con dinero, se suele indicar que el efecto indemnizatorio es restablecer la curva de indiferencia inicial del individuo ${ }^{16}$.

FIG. 3

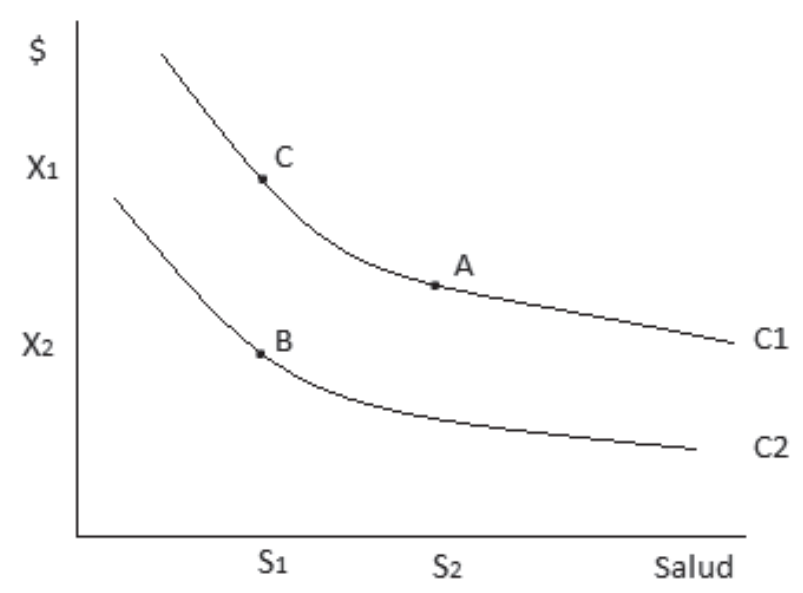

Así, por ejemplo, es posible asumir, como se muestra en la figura 3, que una persona puede encontrarse en un punto A de una curva de indiferencia $(\mathrm{C} 1)$ en el cual posee una combinación de unidades de salud y otro tanto de dinero. El efecto del daño es hacer caer al sujeto dañado a una curva de indiferencia diversa $(\mathrm{C} 2)$ donde podrá tener menos uni-

16 Cooter (2000) 292. 
dades de salud $\left(S_{1}\right)$ y menos también de dinero. El efecto de la indemnización es hacer retornar al individuo a su primitiva curva de indiferencia (C1) aunque no ya en el punto A sino que en el punto $\mathrm{C}$ donde un incremento en el patrimonio $\left(\mathrm{X}_{1}\right)$ viene a compensar tanto la pérdida de patrimonio como la de salud.

Pues bien, esta explicación resulta bastante comprensible cuando la indemnización se impone en un acto único y donde el victimario nada ha hecho para compensar a la víctima luego de acaecido el daño y donde no se contabilizan compensaciones o gastos en que incurren terceros ${ }^{17}$. En un escenario público, no obstante, esta relación compensatoria postaccidente tiende a complejizarse. En efecto, nuestros sistemas jurídicos han generado diversos mecanismos para afrontar los accidentes. El diseño de un sistema sanitario es uno de ellos. A su lado es posible encontrar mecanismos de compensación de seguridad social, beneficios funcionariales que pretenden compensar anticipadamente los riesgos de una determinada actividad o derechamente indemnizaciones pactadas por ley. Este diseńo compensatorio debe insertarse en nuestro modelo compensatorio de tal forma que el aumento patrimonial contemple estas prestaciones pues todas tienen identidad de causa al menos desde una perspectiva económica. Ello, sin embargo, puede presentar el problema de insertar lógicas solidarias en otras de carácter meramente indemnizatorio.

\section{5) EL PROBLEMA DEL MERCADO COMO FOCO DE ANÁlISIS}

El mecanismo compensatorio supone la participación de los diversos agentes en un mercado garantizado por la adopción de opciones racionales por parte de los intervinientes ${ }^{18}$. La racionalidad que se encuentra detrás de esta participación, como hemos indicado, tiene su base en una función de utilidad que a su vez se soporta en aquella maximización de la riqueza propia. La participación en este mercado, además supone capacidades de reacción más o menos rápidas por parte de los intervinientes. En tanto seres maximizadores, las acciones de los intervinientes dependen de las de los demás posibles intervinientes en una relación de riesgos e incentivos. Así las cosas, los gastos en precaución, por un lado, y la concreción de determinados riesgos, por otro, pueden mirarse desde una perspectiva de teoría de juegos. En este escenario, el movimiento hacia escenarios de equilibrio disuade a realizar gastos excesivos en precaución que solo pro-

CANe (2006) 402.

Sobre el supuesto de racionalidad en los mercados económicos resultan útil todas las referencias utilitaristas a la idea de homo aeconomicus. 
vean mayores costos sin generar beneficios adicionales para algunos de los jugadores $^{19}$.

No obstante esto, que viene a confirmar el ideal optimizador de riqueza, su lógica presenta algunos problemas cuando las personas involucradas no buscan la eficiencia de los mismos valores o existe divergencia en la propia medida de valor ${ }^{20}$. En efecto, es un hecho bastante acreditado que el pago de indemnizaciones estatales busca no solo objetivos compensatorios sino que ellas se insertan muchas veces en complejos procesos burocráticos donde intereses comunitarios conviven al lado de aquellos meramente satisfactivos ${ }^{21}$. Por otra parte, a intereses económicos se suelen añadir otro meramente políticos y, en efecto, en términos disuasorios resulta mucho más efectivo transformar costos económicos en costos políticos para lograr una mayor reacción del aparato administrativo ${ }^{22}$. Esta matización, que bien podría considerarse como una objeción grave al análisis de maximización económica, no debiese "paralizar" dicha observación. En vez de ello, debiese servir para analizar la conducta administrativa como una actividad que recoge optimizaciones paralelas. Por un lado económicas, por el otro políticas.

\section{6) EL PROBLEMA DE LA LEGALIDAD}

La adopción de medidas de precaución por parte de privados se desenvuelve dentro de un modelo económico de disposición libre de recursos económicos. En efecto, los particulares no poseen restricciones financieras distintas de su propia capacidad y disposición patrimonial al momento de adoptar sus conductas. En efecto, cuando se opta por una decisión de gasto en la evitación de riesgos el cálculo económico es expresado simplemente en una relación de costo/beneficio.

En el ámbito público en cambio, existe una legalidad dual que regula la actividad administrativa. Por un lado, la Administración puede y debe ejecutar las competencias que una ley le ha asignado, pero por otro solo puede destinar a ello los recursos que otra ley le ha asignado. Un correcto análisis económico de la responsabilidad del Estado debe tomar en consideración este antecedente. Así, o bien presenta una teoría de respaldo de nivel prenormativo que simplemente se aplique a la decisión de política pública de asignación de recursos públicos a las diversas partidas presu-

19 Baird, D. G.; Gertner, R. H. y Picker, R. C. (1994) Game Theory and the Law. Massachusetts: Harvard University Pres, p. 24 y ss.

20 Dworkin, R. (1983). "Why efficiency?” En Kuperberg, M. y Beitz, C. (editores): Law, Economics and Philosophy. New Jersey: Rowman \& Allanheld, p. 123-140.

21 Cohen, D. S. (1990). "Regulating Regulators: The Legal Environment of the State". University of Toronto Law Journal, No 40, p. 231 y ss.

22 Levinson, D. (2000). "Making Government Pay: Markets, Politics, and the Allocation of Constitutional Costs". University of Chicago Law Review, No 67, p. 347. 
puestarias públicas, o bien incluye alguna explicación normativa frente al juzgamiento de la respectiva ley presupuestaria.

\section{3) Conclusiones}

La perspectiva económica de la responsabilidad resulta ser de gran ayuda al momento de analizar la utilidad de ese instrumento como sistema de distribución de riesgo y de localización de recursos económicos. Una genérica optimización de la riqueza es un presupuesto vital de la democracia pues entrega mayores recursos al ámbito de decisión política.

No obstante ello, la aplicación de modelos económicos tiene diversas dificultades en el ámbito público. La introducción de factores políticos en la decisión de adopción de medidas de precaución distorsiona fuertemente la relación funcional de costos y beneficios.

Si se miran los sistemas comparados, es posible percibir que no existe claridad en el ámbito de los fundamentos acerca de cuál es la ratio de los sistemas de responsabilidad del Estado. En Francia, por ejemplo, se ha encontrado en la base ideas tan disímiles como una especie de principio de riesgo de la actividad administrativa, otra de igualdad en las cargas públicas u otra de solidaridad o de equidad ${ }^{23}$. Sin embargo, tal como sostiene Fairgrieve, puede percibirse más una cuestión de énfasis que una directa opción por uno u otro principio ${ }^{24}$. Así, en Francia es posible percibir una opción más determinada por privilegiar un pensamiento más colectivo a la hora de determinar la diligencia a que está obligada la Administración. Ello, sostiene este autor, bien puede estar motivado por una sólida tradición de una línea de pensamiento más colectivista. En Inglaterra, por el contrario, la herencia generada en el rechazo a un derecho desigual para la Administración ha fomentado que sea la idea de "igualdad" la que esté en la matriz del sistema a tal punto que el beneficio para la comunidad es razón suficiente para denegar la responsabilidad llegándose a sostener por la jurisprudencia de forma algo utilitarista que "derechos privados de menor grado deben ceder a un interés público mayor" 25 .

En modelos igualitarios, si es que es posible realmente ser consecuente con ellos, el análisis económico resulta mucho más fructífero y es por ello que el mundo anglosajón ha sido más propenso a su utilización. Por el contrario, en modelos colectivistas, este análisis se complejiza fuertemente al punto de hacer muy poco viable el estudio de la serie de bienes o valores comunes que se persiguen por la vía de una condena indemni-

\footnotetext{
23 Fairgrieve, D. (2003) State Liability in Tort. Oxford: Oxford University Press, p. 265.

24 FAIRGRIEVE (2003) 266.

25 FAIRGRIEVE (2003) 266.
} 
zatoria $^{26}$. Ello, no obstante plantear dudas en su operatividad no niega la premisa principal de todo análisis de políticas públicas y que enseña que antes de discutir sobre derechos es necesario tener el dinero para hacerlos efectivos $^{27}$. Después de todo, como ya sabemos, los derechos cuestan bastante dinero.

\section{4) BibliografíA}

- Abraham, K. S. (2007) The Forms and Functions of Tort Law, $3^{\mathrm{a}}$ edición. New York: Foundation Press.

- Baird, D. G.; Gertner, R. H. y Picker, R. C. (1994) Game Theory and the Law. Massachusetts: Harvard University Pres.

- Barnes, D. W. \& Stout, L. A. (1992) The Economic Analysis of Tort Law. St. Paul: West.

- Belli, P., et al (2001) Economic Analysis of Investment Operations. Washington: World Bank.

- Calabresi, G. (2005) "El costo de los accidentes". En Rosenkrantz, C. (editor): La responsabilidad extracontractual. Barcelona: Gedisa.

- Cane, P. (1997) The anatomy of tort law. Oxford: Hart.

- Cane, P. (2002) "Tort Law as Regulation". Common Law World Review, No 31.

- Cane, P. (2006) Antiyah's accidents, compensation and the law, 7a edición. New York: Cambridge.

- Cohen, D. S. (1990) "Suing the State". University of Toronto Law Journal, No 40.

- Cohen, D. S. (1990) "Regulating Regulators: The Legal Environment of the State". University of Toronto Law Journal, No 40.

- Cooter, R. (1991) "Economic Theories of Legal Liability". The Journal of Economic Perspectives, Vol. 5, No 3.

- Cooter, R. (2000) Law and Economics, 3a edición. Berkeley: Addison Wesley Longman.

- Dworkin, R. (1983) "Why efficiency?" En Kuperberg, M. y Beitz, C. (editores): Law, Economics and Philosophy. New Jersey: Rowman \& Allanheld.

- Fairgrieve, D. (2003) State Liability in Tort. Oxford: Oxford University Press.

- Faure, M. (2009) Tort Law and Economics. Massachusetts: Elgar.

26 Harlow, C. (1996). "State Liability: Problem Without Solution". National Journal of Constitutional Law, Vol. 6, $\mathrm{N}^{\circ}$ 1, p. 68.

27 Holmes, S. y Sunstein, C.R. (1999) The Cost of Rights. Why Liberty Depends on Taxes. New York: Norton. 
- Geistreld, M. (2001) "Economic, Moral Philosophy, and the Positive Analysis of Tort Law”. En Postema, G. J. (editor): Philosophy and the law of torts. New York: Cambridge.

- Harlow, C. (1996) "State Liability: Problem Without Solution". National Journal of Constitutional Law, Vol. 6, $\mathrm{N}^{\circ} 1$.

- Holmes, S. y Sunstein, C.R. (1999) The Cost of Rights. Why Liberty Depends on Taxes. New York: Norton.

- Kuperberg, M. y Beitz, C. (1983) Law, Economics and Philosophy. New Jersey: Rowman \& Allanheld.

- Ogus, A. (2007) "Shifts in Governance for Compensation to Damage: A Framework for Analysis". En Van Boom, W. H. y Faure, M. (editores): Shifts en Compensation between Private and Public Systems. Wien: Springer.

- Perry, S. (2007) "Risk, Harm, Interests and Rights". En Lewens, T. (editor): Risk. Philosophical Perspectives. London: Routledge.

- Posner, R. (1995) "Wealth Maximization and Tort Law: A Philosophical Inquiry”. En Owen, D. G. (editor): Philosophical Foundations of Tort Law. New York: Oxford University Press.

- Vandall, F. (1989) Strict Liability. Legal and Economic Analysis. Connecticut: Greenwood Press. 